\title{
UVES Be observations of early-G dwarfs in old clusters ${ }^{\star}$
}

\author{
S. Randich ${ }^{1}$, F. Primas ${ }^{2}$, L. Pasquini ${ }^{2}$, and R. Pallavicini ${ }^{3}$ \\ 1 INAF/Osservatorio Astrofisico di Arcetri, Largo E. Fermi 5, 50125 Firenze, Italy \\ 2 European Southern Observatory Karl Schwarzschild Strasse 2, 85748 Garching bei München, Germany \\ 3 INAF/Osservatorio Astronomico di Palermo, Piazza del Parlamento 1, 90134 Palermo, Italy
}

Received 10 October 2001 / Accepted 6 March 2002

\begin{abstract}
We have obtained the first beryllium measurements of late F/early G-type stars in the old open cluster M 67 (4.5 Gyr) and in the intermediate age cluster IC 4651 (1.7 Gyr). One member of the young cluster IC 2391 ( $50 \mathrm{Myr}$ ) was also observed. Our sample stars have effective temperatures within a range of $+30-+380 \mathrm{~K}$ from the solar temperature. All our sample stars, including the Sun and the young cluster star have, within the errors, the same Be abundance. This result implies that late F/early G-type stars undergo very little (if any) Be depletion during their main-sequence life-time. Since these stars have undergone some Li depletion, our finding is indicative of shallow mixing, i.e. of a mixing process that can transport surface material deep enough for Li burning to occur, but not deep enough for Be burning. As shown in previous studies, the Li vs. Be diagram is a powerful diagnostic of stellar interiors. In this context, we do not find any evidence of correlated $\mathrm{Li}$ and Be depletion; furthermore, a comparison with various models shows that the Be pattern of our sample stars is compatible only with models including gravity waves. This class of models, however, cannot reproduce the Li observations of M 67 .
\end{abstract}

Key words. stars: abundances - stars: evolution - open clusters and associations: individual: M 67 open clusters and associations: individual: IC 4651

\section{Introduction}

The comparison between the chemical composition of meteorites, assumed to be representative of the primordial solar cloud, and the Sun shows that lithium is 140 times underabundant in the solar photosphere (Anders \& Grevesse 1989). This result cannot be explained as due to the action of convection only since, according to standard stellar models, the convective zone of solar type stars on the main sequence (MS) does not reach deep enough layers to enable lithium destruction (e.g., Pinsonneault 1997). Besides Li depletion in the Sun, several pieces of observational evidence have been found indicating that, in contrast to standard models, late F/early G-type stars do deplete Li while on the MS (e.g., Jeffries 2000, Pasquini 2000 and references therein), witnessing the action of an additional process that can transport material from the surface to the stellar interior. Whereas several models have been developed, which take into account a more complex physics,

Send offprint requests to: S. Randich,

e-mail: randich@arcetri.astro.it

* Based on observations collected at European Southern Observatory, Chile (65.L-0427). Part of the data were obtained as part of an ESO Service Mode run. including mass loss, diffusion, slow mixing driven by rotation or gravity waves (Swenson \& Faulkner 1992; Chaboyer et al. 1995; Deliyannis \& Pinsonneault 1997; Montalbán \& Schatzman 2000), these models are still very poorly constrained; in particular, the question remains how deep in the stellar interior the mixing extends.

Since beryllium burns at temperatures $10^{6} \mathrm{~K}$ higher than $\mathrm{Li}(\mathrm{Li}$ and $\mathrm{Be}$ are destroyed by proton capture at the temperatures of 2.5 and $3.5 \times 10^{6} \mathrm{~K}$, respectively) the simultaneous determination of $\mathrm{Li}$ and Be abundances in the same star traces stellar mixing to different depths, thus providing stringent observational constraints on model predictions. Whereas until recently it was commonly accepted that the Sun had a Be abundance a factor of about two lower than meteoritic, Balachandran \& Bell (1998) carried out a revised analysis of the solar spectrum in the near-UV and found a solar Be abundance $\log n(\mathrm{Be})_{\odot}=1.40$, thus comparable with the meteoritic value $\log n(\mathrm{Be})=1.42$ (Anders \& Grevesse 1989). They concluded that Be is undepleted in the solar photosphere and suggested that mixing in the solar interior is shallower than previously thought. The result of Balachandran \& Bell however is still controversial (e.g., King et al. 1997); in particular, the finding of little or no Be depletion in 
the Sun cannot be regarded as a general conclusion, since it relies on absolute abundance measurements, which are hampered by large uncertainties. Furthermore, we do not know whether and to what extent our Sun is representative of the whole population of stars with similar mass and evolutionary status. Finally, according to most of the proposed models, mixing depends on the whole evolutionary history of a star: hence, knowledge of the Be abundance for only one star at the age of the Sun does not allow discrimination between different models, since stars with different parameter evolution (e.g. rotation) may undergo different amounts of mixing. Beryllium measurements in homogeneous samples of stars, such as stellar clusters, with well determined evolutionary status, chemical composition, and age, are thus crucial. In addition, since most models predict significant Be variations on long timescales (>1 Gyr), a meaningful comparison between model predictions and observations should include old stars.

The Be feature that is best observed, the Be II resonance doublet $(\lambda=3130.420 \AA$ and $\lambda=3131.064 \AA)$, lies close to the atmospheric UV cutoff; observations of Be are thus very challenging and have so far been limited to the brightest stars observable in the field (e.g., Primas et al. 2001) and in the close-by Hyades and Ursa Major clusters (Boesgaard \& Budge 1989; García López et al. 1995; Boesgaard \& King 2002).

Thanks to the superior near-UV capabilities of UVES on VLT Kueyen (Dekker et al. 2000), we were able to acquire for the first time high resolution spectra of the Be region of 14 th magnitude $\mathrm{G}$ dwarfs in old clusters. In the present paper we report the results concerning $\mathrm{Li}$ and Be, while an abundance analysis including heavy elements will be presented in a forthcoming paper.

\section{Observations}

Our sample includes five members of the solar age M 67 cluster (4.5 Gyr), three members of the 1.7 Gyr old IC 4651, the solar-analog $\alpha$ Cen A, and one member of the young (50 Myr) cluster IC 2391. This star has just arrived on the zero age main sequence and it is undepleted in $\mathrm{Li}$ (Randich et al. 2001); under the fully reasonable assumption that it is also undepleted in Be, it can be used to set a zero point to our abundance scale and it may provide an indication of the initial Be abundance. No known binaries are included in our sample.

The observations were carried out using UVES on VLT UT2 (Kueyen). M 67 members and $\alpha$ Cen A were observed in Visitor Mode in April 2000; the young cluster member was also observed in Visitor Mode in Feb. 2001, as part of a different program; finally, the three targets in IC 4651 were instead observed in Service Mode during March 2000. All the stars were observed using the same set-up of UVES; namely, UVES was operated in Dichroic Mode using Cross Dispersers \#1 and \#3 in the Blue and Red arms, respectively. The Blue arm is equipped with a EEV $2048 \times 4102$ CCD, while the Red arm is equipped with a mosaic of two CCDs made by a EEV
$2048 \times 4102$ CCD and a MIT-LL $2048 \times 4102$ CCD. Such a combination allowed us to cover the spectral ranges from $\sim 3115$ to $3940 \AA$ in the blue and from $\sim 4780$ to $6810 \AA$ in the red. The $15 \mu \mathrm{m}$ pixels and the use of a 1 arcsec wide slit (projecting into 4 pixels) and CCD binning $2 \times 2$ and $1 \times 1$ in the blue and red, respectively, yielded resolving powers $R \sim 40000$ and $R \sim 45000$. The spectra were reduced using MIDAS and following the usual steps: bias and interorder background were subtracted from both science and flat-field frames; the science frames were then optimally extracted, flat-fielded, and wavelength calibrated. Finally, the extracted orders were merged. The exposure times of cluster stars ranged between $40 \mathrm{~min}$ and $3 \mathrm{hrs}$; the sample stars were observed under different seeing conditions and variable airmasses, due to the long exposure times. The final $S / N$ ratios per spectral bin are comprised between $\sim 30$ and 50 in the Be region and between $\sim 100$ and 200 in the Li region. A much higher $S / N$ was achieved for $\alpha$ Cen $\mathrm{A}$. The sample stars together with details on the observations are listed in Table 1 . In Fig. 1 we show the comparison of the spectra of two M 67 members in the Be and Li spectral regions.

\section{Abundance and error analysis}

Effective temperatures were determined based on $B-$ $V$ colors using the same calibration employed in similar studies (Soderblom et al. 1993). A surface gravity $\log g=4.44$ and microturbulence $\xi=1.1 \mathrm{~km} \mathrm{~s}^{-1}$ were assumed for all sample stars. Rotational velocities were retrieved from Pasquini et al. (in preparation) for M 67 and IC 4651 and from Randich et al. (2001) for the young cluster member. $T_{\text {eff }}$ and $v \sin i$ values are listed in Cols. 2 and 3 of Table 2 .

$\mathrm{Li}$ and Be abundances were derived in a consistent way using Kurucz model atmospheres and codes (ATLAS9, SYNTHE, and WIDTH9 - Kurucz 1993). Model atmospheres were computed for the exact stellar parameters of each star, by interpolating in the ATLAS9 grid of models officially released by Kurucz on CD-ROMs (Kurucz 1993). Solar abundances were taken from Anders \& Grevesse (1989).

Be abundances were determined by spectrum synthesis using the line list extensively tested by Primas et al. (1997). This compilation has been constrained by using a large sample of stars, and after having run several tests on the possible presence of unidentified lines in the Be II doublet region. The main reason behind these tests (also carried out by similar studies) is the following: using the list of atomic and molecular lines derived from laboratory measurements only and model atmospheres currently available, it is very difficult to obtain a satisfactory fit of the $\lambda 3131.064 \AA$ line with the solar photospheric abundance $\left(\log n(\mathrm{Be})_{\odot}=1.15\right.$, as determined by Chmielewski et al. 1975). Possible ways out include the introduction of one or more (still unidentified, hence "predicted") lines or adjusting the oscillator strengths of neighbouring lines (cf. García López et al. 1995). Though different, these two 
Table 1. Sample stars and Log of the observations.

\begin{tabular}{|c|c|c|c|c|c|c|}
\hline star & $V$ & $(B-V)_{0}$ & $\begin{array}{l}\text { Night } \\
\text { (UT) }\end{array}$ & $\begin{array}{l}\text { Exp. } \\
\text { (s) }\end{array}$ & $S / N @ \mathrm{Be}$ & $S / N @ \mathrm{Li}$ \\
\hline$\alpha$ Cen A & -0.1 & 0.63 & 2000 Apr. 11 & $4(\mathrm{~b}) / 2(\mathrm{r})$ & 120 & 340 \\
\hline IC 2391 SHJM2 & 10.3 & 0.57 & 2001 Feb. 16 & 2400 & 30 & 210 \\
\hline M 67 S988 & 13.18 & 0.534 & 2000 Apr. 15 & 6300 & 40 & 120 \\
\hline M 67 S994 & 13.18 & 0.535 & 2000 Apr. 14 & 10800 & 50 & 160 \\
\hline M 67 S1252 & 14.07 & 0.587 & 2000 Apr. 16 & 5400 & 40 & 110 \\
\hline M 67 S1256 & 13.67 & 0.595 & 2000 Apr. 15 & 9100 & 45 & 120 \\
\hline M 67 S969 & 14.18 & 0.622 & 2000 Apr. 16 & 5400 & 40 & 110 \\
\hline IC 4651 EG7 & 14.20 & 0.557 & 2000 Mar. 26 & 14400 & 45 & 180 \\
\hline IC 4651 EG45 & 14.20 & 0.568 & 2000 Mar. 29 & 10800 & 40 & 140 \\
\hline IC 4651 AT2105 & 14.02 & 0.545 & 2000 Mar. 30 & 9540 & 40 & 140 \\
\hline
\end{tabular}

Numbering for M 67 from Sanders (1977).

Numbering for IC 4651 from Eggen (1971) and Anthony-Twarog et al. (1988).

approaches give similar results, a satisfactory fit of the solar spectrum with the abundance $\log n(\mathrm{Be})_{\odot}=1.15$. For our analysis, we adopted the line list from Primas et al. (1997), including a predicted Fe I line at $3131.043 \AA$, the strength of which had been constrained by using a large sample of stars of different $T_{\text {eff }}, \log g$, and spanning three orders of magnitude in metallicity (cf. the original work for more details).

We started our analysis from fitting the Kurucz Solar Flux Atlas (Kurucz et al. 1984): using a solar model with $T_{\text {eff } \odot}=5777 \mathrm{~K}, \log g_{\odot}=4.437, \xi_{\odot}=1.1 \mathrm{~km} \mathrm{~s}^{-1}$, we obtained a solar Be abundance $\log n(\mathrm{Be}) \odot=1.11$, in very good agreement with the value found by Chmielewski et al. (1975). To gain confidence on the robustness of our analytical method, we then fitted our own spectrum of the solar-analog $\alpha$ Cen A $\left(T_{\text {eff }}=5800 \mathrm{~K}\right.$, $\log g=4.44,[\mathrm{M} / \mathrm{H}]=+0.1$, cf. Primas et al. 1997). We found $\log n(\mathrm{Be})_{\odot}=1.17$, in good agreement with the Sun and with values derived from previous analyses (within the quoted uncertainties - cf. Primas et al. 1997; King et al. 1997). Then, we applied the same technique to all our sample stars, including also the member of the young cluster IC 2391. Examples of some spectrum synthesis fits are shown in Figs. 2-5, while all the Be (derived by demanding a satisfactory fit of both $\mathrm{Be}$ lines) and $\mathrm{Li}$ abundances are reported in Table 2, together with their respective uncertainties. For the records, using model atmospheres computed with the overshooting option switched off (NOVER) translates into a systematic difference $\Delta \log n(\mathrm{Be})=-0.05$ dex for all stars.

$\mathrm{Li}$ abundances were derived from the measured equivalent widths of the Li I $6707.8 \AA$ feature (see Table 2). Given the resolution of our spectra and the rotational velocity of the sample stars, the Li feature was not blended with the nearby Fe I $6707.44 \AA$ line and, thus, we did not need to correct the measured $\mathrm{Li} E W \mathrm{~s}$ for the contribution of the latter. For completeness and consistency, Li abundances for stars from previous studies (namely, Jones et al. 1999 for M 67 and Randich et al. 2000 for IC 4651) have also been re-computed from the $E W$ s published in the original works. We note that new abundances are in very good agreement with old ones.

Errors in the derived abundances were computed from analyzing the dependence of $\mathrm{Li}$ and $\mathrm{Be}$ abundances to changes in the adopted stellar parameters. Uncertainties of $\pm 100 \mathrm{~K}$ in $T_{\text {eff }}, 0.2$ dex in log g, and $0.2 \mathrm{~km} \mathrm{~s}^{-1}$ in $\xi$ were assumed. The final uncertainties listed in Table 2 include also the sensitivity of Be to the placement of the continuum (estimated to be on the order of $\pm 3 \%$ ) and of $\mathrm{Li}$ to errors in the measured $E W \mathrm{~s}$. There may be extra sources of systematic and random errors. In the case of Be, one may want to take into account extra uncertainties encountered during spectrum synthesis. During our analysis we have established that including one "predicted" line (or adjusting the oscillator strengths of one or more lines) implies a systematic difference of $\sim-0.05$ dex for our sample stars here (as to say, without the predicted line, one has to increase the Be abundance by +0.05 dex in order to get a similar satisfactory fit). Another way is to run a synthesis including the entire Kurucz list of "predicted" (as opposed to "laboratory") lines, in order to quantify the global effect on the continuum. Although related to the same problem, this may be considered a random source of error. We found it affects the continuum level by $\sim 3 \%$, which corresponds to \pm 0.05 dex in beryllium. Another weak point to be aware of is the well known discrepancy between the solar photospheric $\mathrm{Be}\left(\log n(\mathrm{Be})_{\odot}=1.15\right)$ and the abundance measured in meteorites $\left(\log n(\mathrm{Be})_{\odot}=1.42\right.$ determined by Anders \& Grevesse 1989). Though this may turn 

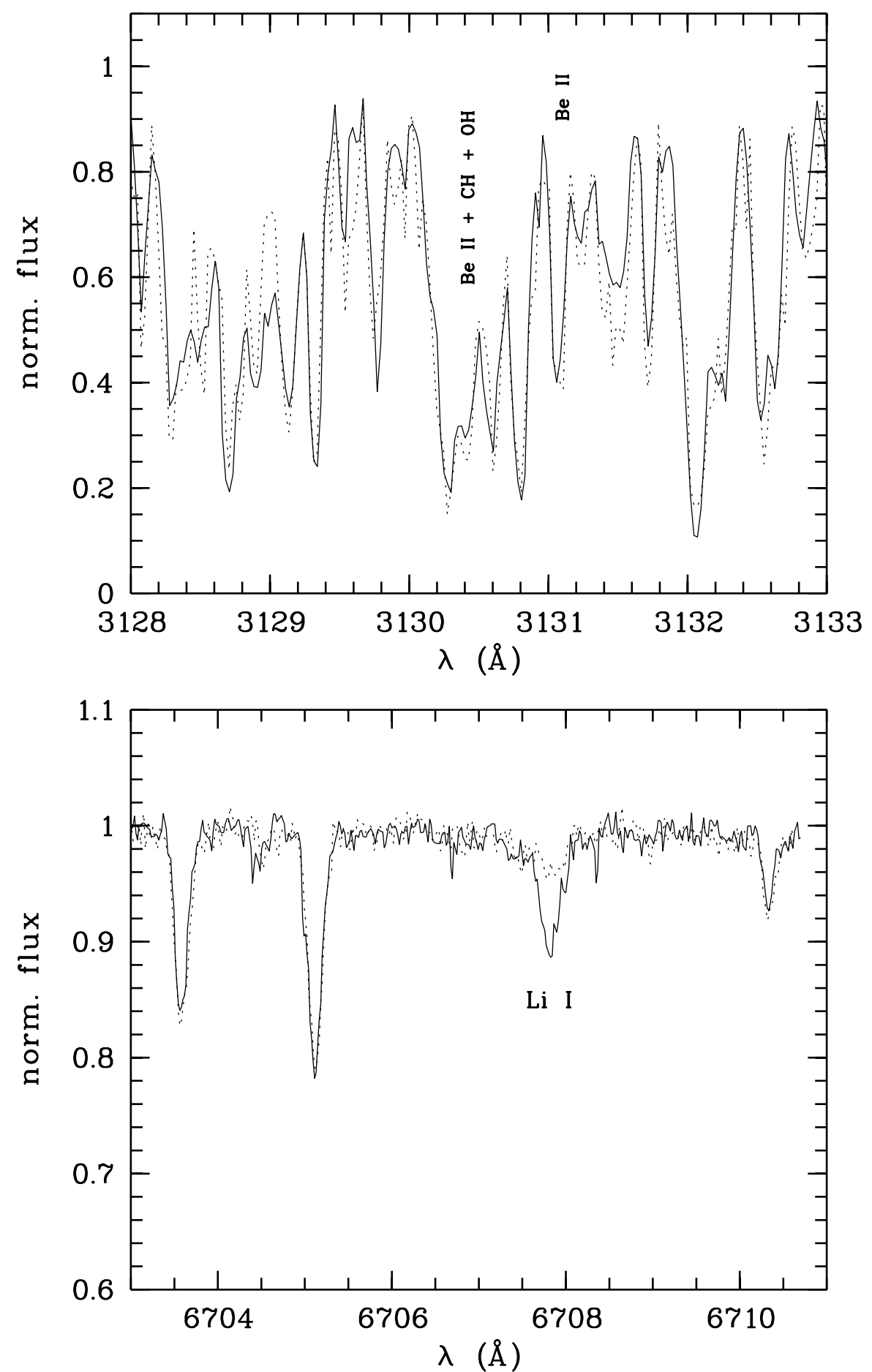

Fig. 1. Comparison of the spectra in the Be (top panel) and Li (bottom panel) spectral regions of the stars S1252 (solid line) and S1256 (dashed line) in the old cluster M 67. The figure clearly shows the lack of correlated Li and Be depletion; whereas a large difference in Li strengths is evident, the spectra in the Be region do not show a significant disagreement. Li equivalent widths of 33 and $11 \mathrm{~m} \AA$ were measured for S1252 and S1256 respectively, resulting in a factor of $\simeq 5$ difference in Li abundance.

out to be only an apparent discrepancy (cf. Balachandran \& Bell 1998, who determined a solar photospheric Be in very good agreement with the meteoritic value by including new continuum opacity sources), we decided not to enter the discussion which, as mentioned above, is still very much open, as also shown by Allende Prieto \& Lambert (2000) who find that there is no missing UV opacity.
In conclusion, based on the quality of our observations and on the fact that our main conclusions will be based on differential comparison between stars characterized by similar parameters, we believe that the relative error bars quoted in Table 2 are appropriate. In other words, thanks to our choice of targets of very similar nature and macroscopic parameters, we can safely compare the relative light 


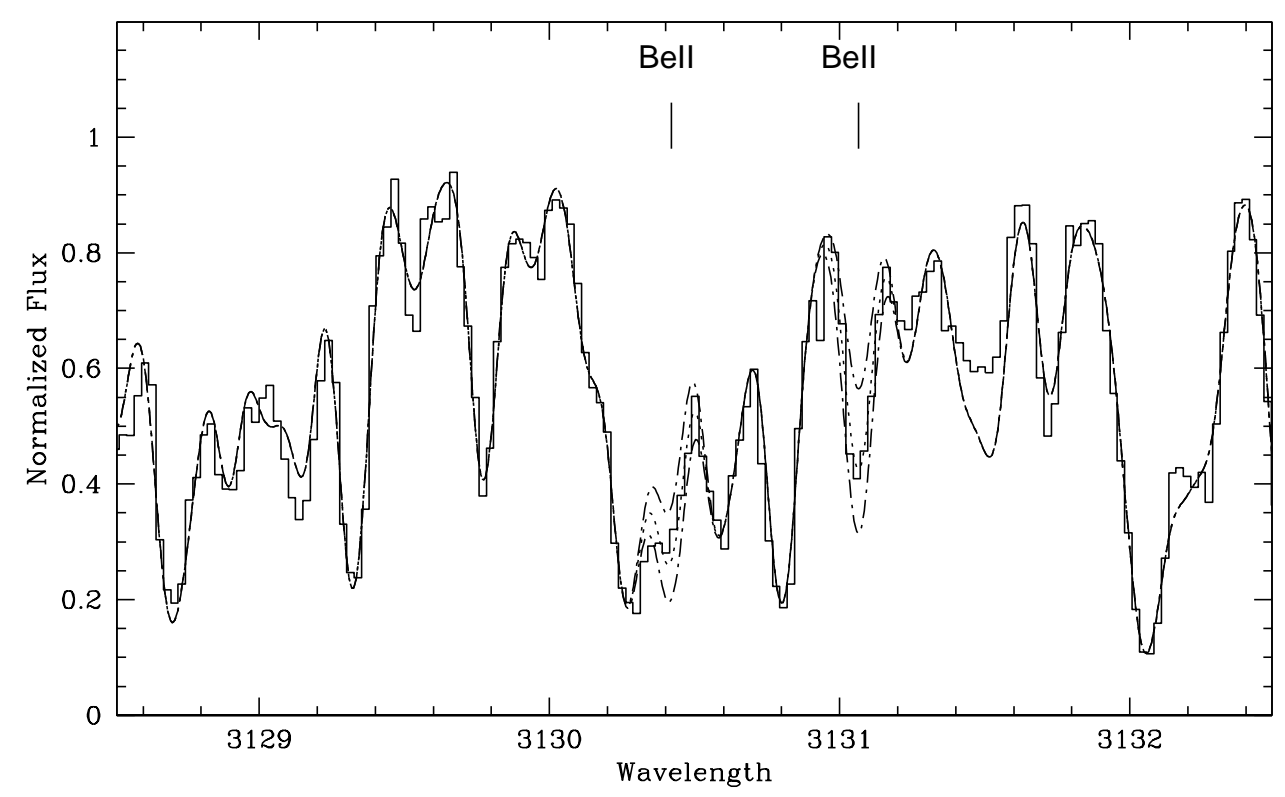

Fig. 2. The spectrum synthesis fit for the star S1252 in M 67. The histogram represents the observed spectrum; the dotted line denotes the best fit, while the two dot-dashed lines indicate synthesis obtained with a factor of two lower/higher Be abundance.

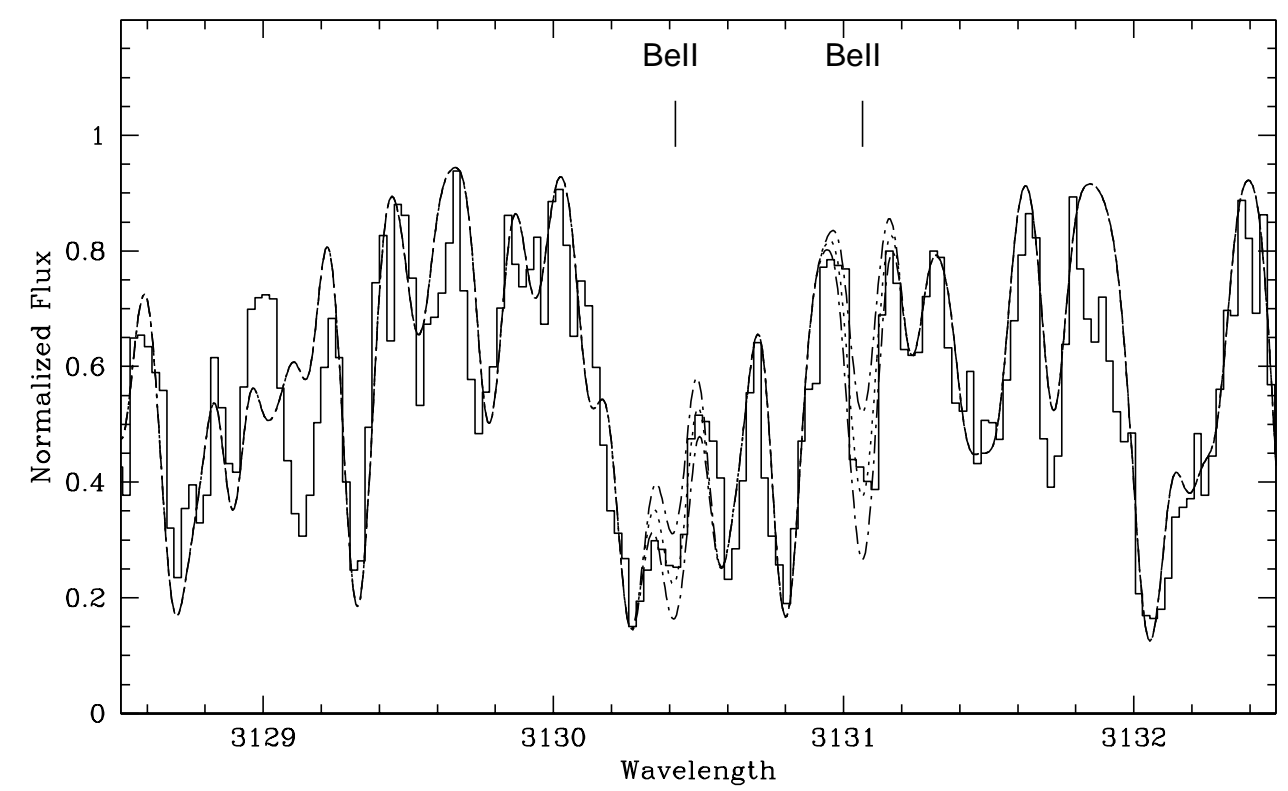

Fig. 3. Same as Fig. 2, but the spectrum synthesis for S1256 in M 67 is shown.

element abundances of our sample stars without being affected by systematic effects. This implies that differences in Be abundances are better constrained than absolute abundances.

\section{Results and discussion}

\subsection{Be and Li abundances}

In Fig. 6 we show $\log n(\mathrm{Be})$ vs. $T_{\text {eff }}$ for our sample stars. The Sun is also included in the figure. Figure 6 and Table 2 clearly show that all our sample stars have, within the errors, the same Be abundances: in particular, we find for the Sun exactly the same abundance as for the $50 \mathrm{Myr}$ old IC 2391 member; stars in M 67 (with the possible exception of S988 that shows a somewhat lower abundance), IC 4651, and $\alpha$ Cen $\mathrm{A}$ also have a similar abundance as the Sun. We obtain an average abundance $\log n(\mathrm{Be})=$ $1.08 \pm 0.12$ for $\mathrm{M} 67$ and $\log n(\mathrm{Be})=1.13 \pm 0.03$ for IC 4651 . We stress that the finding of similar abundances is extremely robust since it is based both on direct comparison of the spectra and on a consistent analysis of stars with very similar macroscopic parameters.

As we have mentioned, the Be abundance that we derive for the Sun is in agreement with the value $\log n(\mathrm{Be})=$ 1.15 derived by Chmielewski et al. (1975); this value, as well the abundances of our sample stars, are about a factor 


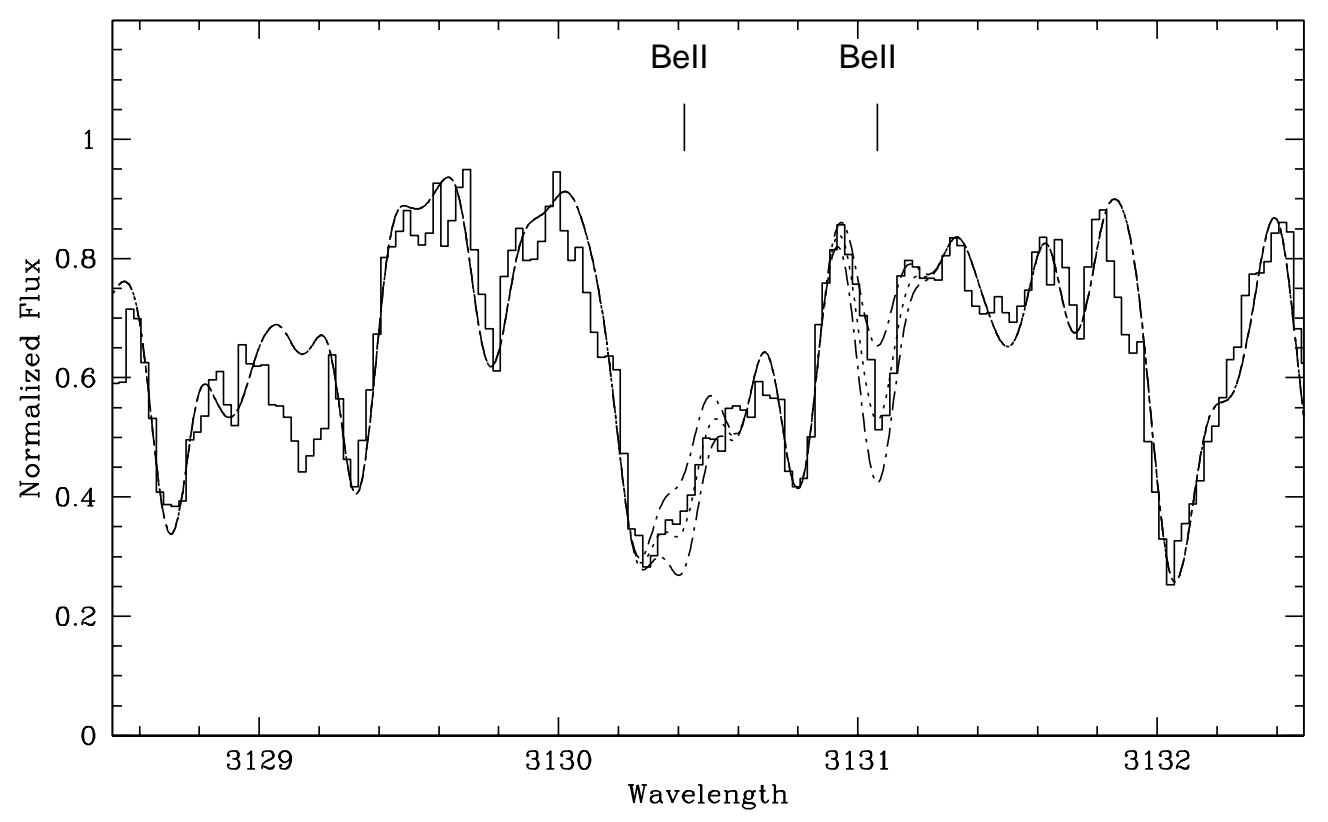

Fig. 4. Same as Fig. 2, but the spectrum synthesis for AT2105 in IC 4651 is shown.

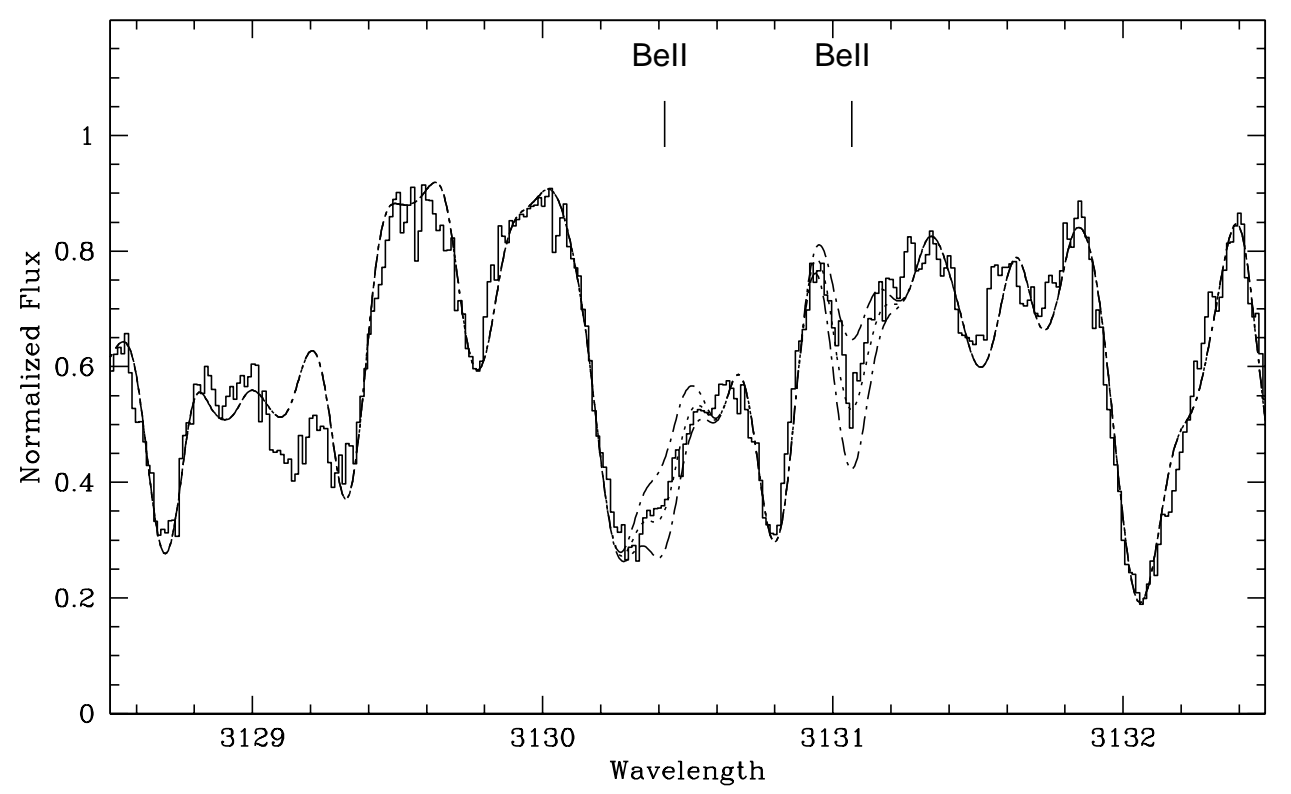

Fig. 5. Same as Fig. 2, but the spectrum synthesis for the star in the young clusters IC 2391 is shown.

of two lower than the meteoritic abundance $\log n(\mathrm{Be})=$ 1.42. For the three clusters we have derived similar, about solar, metallicity (see also Randich et al. 2001): given the $\log n(\mathrm{Be})$ vs. $[\mathrm{Fe} / \mathrm{H}]$ relationship (e.g., Boesgaard et al 1999), this suggests a similar initial Be abundance. Two hypotheses can be proposed to explain the difference between the abundances that we have derived for our sample stars and the meteoritic Be abundance: a) All our sample stars, including the young cluster star, have undergone a very similar amount of Be depletion; b) The difference between our best fit Be abundances and the meteoritic value is simply due to different analytical methods adopted during the analyses. Under this hypothesis, none of our sam- ple stars has undergone any Be depletion, implying that stars in this temperature range including the Sun, do not burn Be while on the MS. Hypothesis a) would mean that some Be burning occurs during the pre-main sequence (PMS) phases, in contradiction with all model predictions (e.g., Piau \& Turck-Chieze 2002) and inconsistently with the observational evidence of no PMS Li depletion for stars in this $T_{\text {eff }}$ range (e.g., Randich et al. 2001 and references therein); in other words, the simultaneous Be depletion and Li non-depletion in the young star would be hardly explainable. For these reasons, we regard hypothesis a) as very unlikely. Hypothesis b) would instead be the proof of a shallow mixing process, i.e. a mechanism that is able to 


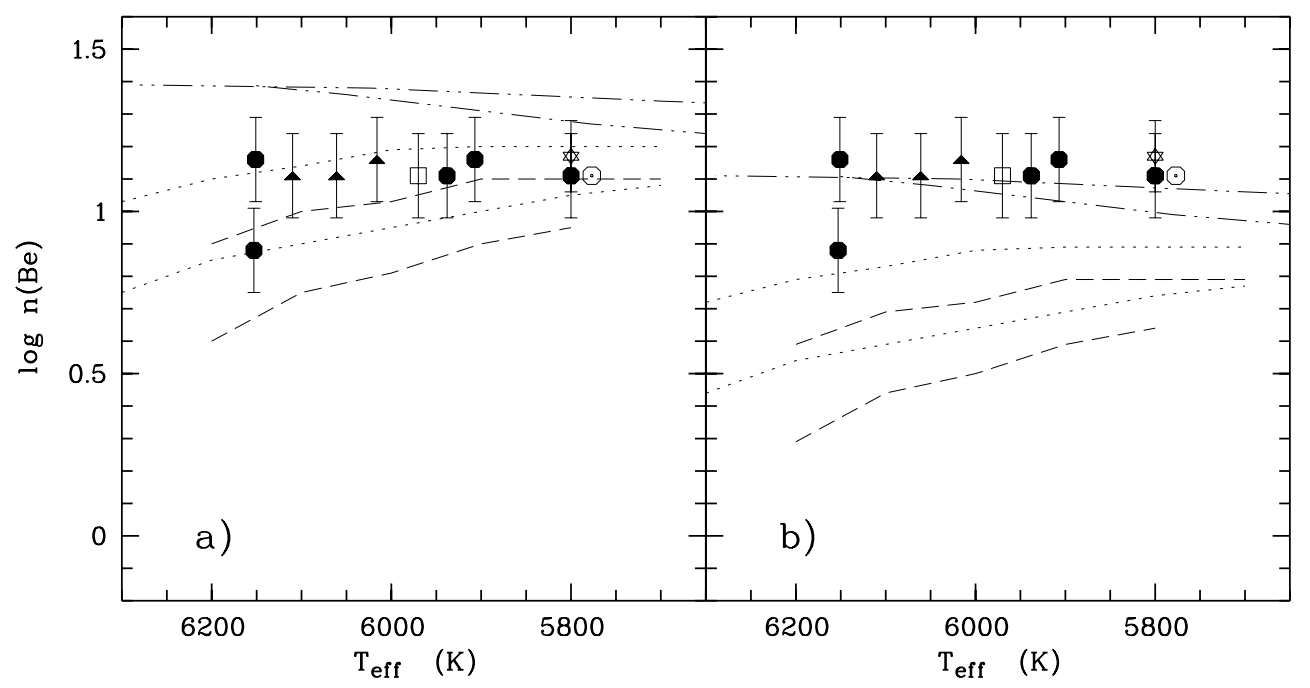

Fig. 6. $\log n(\mathrm{Be})$ vs. effective temperature for our sample stars. Filled circles and triangles denote M 67 and IC 4651 members, respectively, while the open square indicates the young cluster member. The Sun (Sun symbol) and $\alpha$ Cen A (star symbol) are included in the figure. Curves denoting model predictions are shown in the figure. Dot-dashed curves indicate the predictions of models including gravity waves, with upper and lower curves denoting predictions at an age of 1.7 and 4.0 Gyr, respectively. Dotted and dashed curves indicate the predictions of rotationally induced mixing at 1.7 and 4 Gyr; for each curve style, upper curves refer to models with an initial rotational velocity of $10 \mathrm{~km} \mathrm{~s}^{-1}$, while lower curves denote the predictions of models with an initial rotational velocity of $30 \mathrm{kms}^{-1}$. In panel a) theoretical curves are normalized to an initial abundance equal to the meteoritic value $\log n(\mathrm{Be})=1.42$, while in panel $\mathbf{b}$ ) the curves are normalized to the abundance $\log n(\mathrm{Be})=1.11$ that we derived for the young cluster member.

Table 2. Effective temperatures, rotational velocities, and derived abundances.

\begin{tabular}{lccccc}
\hline \hline star & $\begin{array}{c}T_{\text {eff }} \\
(\mathrm{K})\end{array}$ & $\begin{array}{c}v \sin i \\
\left(\mathrm{~km} \mathrm{~s}^{-1}\right)\end{array}$ & $\begin{array}{c}E W(\mathrm{Li}) \\
(\mathrm{mA})\end{array}$ & $\log n(\mathrm{Li})$ & $\log n(\mathrm{Be})$ \\
\hline$\alpha$ Cen A & 5800 & 2 & $9 \pm 0.5$ & $1.24 \pm 0.11$ & $1.17 \pm 0.11$ \\
IC 2391 SHJM2 & 5970 & 9 & $150 \pm 3$ & $3.45 \pm 0.09$ & $1.11 \pm 0.13$ \\
M 67 S988 & 6153 & 6 & $12 \pm 2$ & $1.89 \pm 0.13$ & $0.88 \pm 0.13$ \\
M 67 S994 & 6151 & 6 & $25 \pm 2$ & $2.27 \pm 0.11$ & $1.16 \pm 0.13$ \\
M 67 S1252 & 5938 & 4 & $33 \pm 3$ & $2.35 \pm 0.11$ & $1.11 \pm 0.13$ \\
M 67 S1256 & 5907 & 4 & $11 \pm 1$ & $1.60 \pm 0.11$ & $1.16 \pm 0.13$ \\
M 67 S969 & 5800 & 4 & $28 \pm 2$ & $2.06 \pm 0.10$ & $1.11 \pm 0.13$ \\
IC 4651 EG7 & 6061 & 4 & $52 \pm 3$ & $2.61 \pm 0.11$ & $1.11 \pm 0.13$ \\
IC 4651 EG45 & 6016 & 4 & $48 \pm 3$ & $2.52 \pm 0.11$ & $1.16 \pm 0.13$ \\
IC 4651 AT2105 & 6110 & 8 & $67 \pm 6$ & $2.82 \pm 0.12$ & $1.11 \pm 0.13$ \\
\hline
\end{tabular}

transport surface material down to the Li burning layer, but not deep enough to destroy Be.

In Fig. 7 we plot $\log n(\mathrm{Li})$ vs. $T_{\text {eff }}$ for our sample stars and the stars studied by Jones et al. (1999) and Randich et al. (2000). The present study confirms the star-to-star scatter in Li among M 67 members (Spite et al. 1987; García López et al. 1988; Pasquini et al. 1997; Jones et al. 1999), indicating different amounts of $\mathrm{Li}$ depletion for stars with the same mass and chemical composition. In agreement with previous studies (Randich et al. 2000), stars in the younger IC 4651 cluster do not show any major dispersion, suggesting that the mechanism responsible for the dispersion observed in M 67 acts at relatively old ages.

\subsection{Be vs. Li depletion and comparison with the models}

As clearly pointed out by Deliyannis (2000), the $\log n(\mathrm{Be})$ vs. $\log n(\mathrm{Li})$ diagram is a very powerful tool to investigate the nature of stellar mixing and to discriminate between different models. It is worth emphasizing that in this diagram the shape of the relationship, i.e., the relative $\mathrm{Li}$ and $\mathrm{Be}$ abundances, rather than absolute 


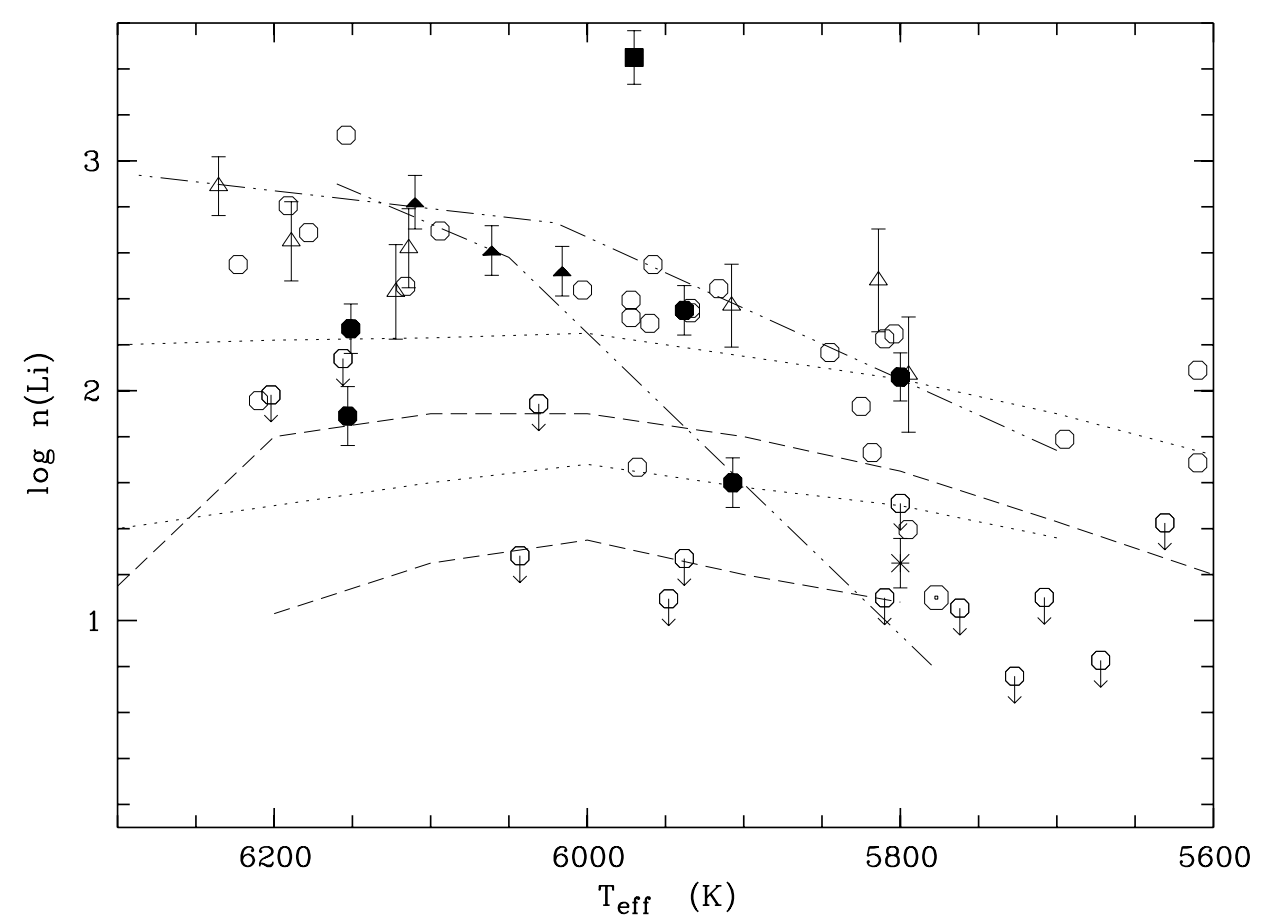

Fig. 7. $\log n(\mathrm{Li})$ vs. effective temperature for our sample stars. Symbols and curves are the same as in Fig. 6, but filled symbols refer to present data (namely, filled circles: M 67; filled triangles: IC 4651; filled square: young star; asterisk: $\alpha$ Cen A), while open circles and triangles denote Li abundances for M 67 and IC 4651 from previous studies (Jones et al. 1999 and Randich et al. 2000).

abundances, is providing most of the information. In Fig. 8 we show $\log n(\mathrm{Be})$ vs. $\log n(\mathrm{Li})$ for our sample stars and the Sun; the best fit relationship between $\log n(\mathrm{Be})$ and $\log n(\mathrm{Li})$ proposed by Boesgaard et al. (2001) for field stars with $6300 \geq T_{\text {eff }} \geq 5850 \mathrm{~K}$ is also shown in the figure.

Be surveys among field stars (e.g., Stephens et al. 1997; Boesgaard et al. 2001) have reported the existence of a correlation between $\mathrm{Li}$ and Be depletion; this correlation was interpreted as the proof of the action of slow mixing driven by rotation; a similar result was found by Boesgaard \& King (2002) for Hyades dwarfs warmer than $5850 \mathrm{~K}$. At odds with the correlation found for field stars, and in contrast with the prediction of slow mixing induced by rotation (see e.g., Deliyannis 2000), Fig. 8 does not show any clear evidence of correlated $\mathrm{Li}$ and Be depletion; stars that have suffered different amounts of Li destruction both in the same cluster and in different clusters, have, within the errors, the same Be content (see also Fig. 6). Our datapoints plotted in Fig. 8 do not follow the mean trend found for field stars with $6300 \geq T_{\text {eff }} \geq 5850 \mathrm{~K}$. This is not unexpected for the stars in our sample with $T_{\text {eff }}$ below $5850 \mathrm{~K}$ (namely, the Sun, alpha Cen A, and S969 in $\mathrm{M}$ 67), for which no correlation between $\mathrm{Li}$ and $\mathrm{Be}$ is indeed found for the field stars. It is instead surprising that the warmest stars in our sample do not follow the same relationship as field stars. Most of the stars in M 67 and IC 4651 are only marginally in agreement with the relationship for field stars, with star S1256 in M 67 clearly showing much less Be depletion than expected; viceversa, the young cluster star has a Be abundance significantly below the expected value. If one changed the intercept of the curve to fit this star, all the other sample stars would then lie much above the relationship. We mention that different temperatures were reported in the literature for S1256, a couple of which are cooler than $5850 \mathrm{~K}$. More in general, our temperature scale is different from that of Boesgaard et al. (2001) and thus other stars in our sample, besides $\alpha$ Cen A, the Sun, and S969, might fall outside the interval $5850 \leq T_{\text {eff }} \leq 6300 \mathrm{~K}$ based on the calibration of Boesgaard et al. In order to further investigate this point, we re-determined the effective temperatures of field stars in the sample of Boesgaard et al. using our calibration. Whereas we do find differences as large as $\sim 250 \mathrm{~K}$, we do not find a systematic offset and the range $5850-6300 \mathrm{~K}$ of Boesgaard et al. reflects into an interval of 5810-6480 K in our scale. In other words, given the uncertainties in the temperature scales, the lower limit for the Li-Be correlation found for field stars cannot be defined in an absolute way. On the other hand, the discrepancy between the correlation found for field stars and the lack of correlation found for our sample stars cannot be explained as due to different temperature calibrations.

In the following, our results are compared with the predictions of various models.

Models including diffusion actually do not warrant a detailed discussion: as discussed by Deliyannis (2000) or by Stephens et al. (1997), diffusion implies simultaneous $\mathrm{Li}$ and Be depletion and thus does not fit the observed Be vs. Li diagram shown in Fig. 8. In Figs. 6 and 7, 


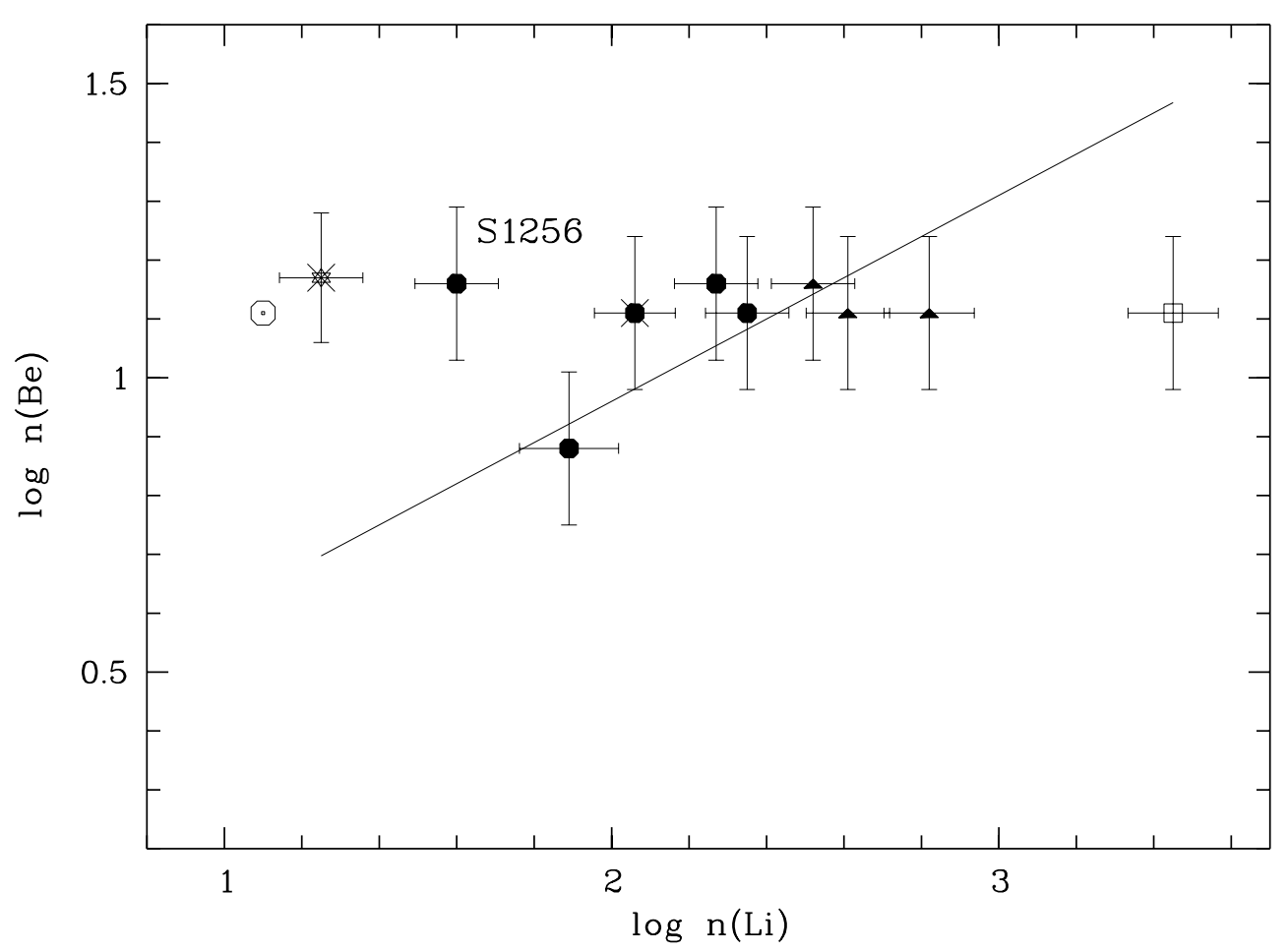

Fig. 8. $\log n(\mathrm{Be})$ vs. $\log n(\mathrm{Li})$. Circles and triangles indicate M 67 and IC 4651 members, respectively; the Sun is denoted by the Sun symbol, $\alpha$ Cen A by a star symbol, and the young star in IC 2391 by an open square. Crossed symbols denote stars with $T_{\text {eff }}$ below $5850 \mathrm{~K}$. The solid line indicate the best fit relationship found by Boesgaard et al. (2001) for field stars with $T_{\text {eff }}$ in the interval 5850-6300 K.

instead, we show a quantitative comparison with the predictions of models including slow mixing processes; more specifically, we plot different curves denoting theoretical predictions by models including slow rotationally induced mixing (retrieved from Deliyannis \& Pinsonneault 1997) and by models including gravity waves (kindly provided by J. Montalbán - see also Montalbán \& Schatzman 2000). Following the two hypothesis a) and b) discussed in the previous section, we show in panel a) of Fig. 6 model predictions with an initial Be abundance equal to the meteoritic value, as assumed in the original works presenting these models. In panel b) the curves are instead normalized to an initial abundance $\log n(\mathrm{Be})=1.11$ as derived by us for the young, Li-undepleted star in our sample; under the assumption that this star has not depleted any Be, its abundance is indicative of the initial Be abundance in our abundance scale.

Models including rotational mixing predict that some Be depletion has occurred already at 1.7 Gyr and that additional depletion should take place between 1.7 Gyr and the solar age. In addition, at a given age, they predict a difference in both $\log n(\mathrm{Li})$ and $\log n(\mathrm{Be})$ for stars with the same temperature, but different initial rotational velocities. The difference in $\mathrm{Li}$ abundance is much larger than the difference in Be abundance. In other words, according to these models, some dispersion in Be should be observed if cluster stars had different initial rotation rates. A larger dispersion in $\mathrm{Li}$ is predicted.
The absolute Be abundances of M 67 and IC 4651 members shown in panel a) are, within the errors, in fairly good agreement with the predictions by rotational mixing. However, as evidenced in Sect. 4.1, the young cluster member exhibits an abundance which is a factor $\sim 2$ below meteoritic. The Be content of this star indeed appears in agreement with the predicted Be abundance at an age between 1.7 and $4.5 \mathrm{Gyr}$, which is clearly inconsistent with the much younger age of the star. Panel b) has been made under the assumption that the Be abundance of the young star would represent the initial Be abundance; therefore, and by definition, this panel does not evidence any inconsistency for the young cluster member. On the contrary, the observed Be abundances of both M 67 and IC 4651 members are not fitted by the models including rotational mixing that predict larger depletion than observed. Only the warmest star in our sample, S988 in M 67, is marginally in agreement with model predictions. Independently from the initial absolute Be abundance, rotational mixing is not able to reproduce the relative $\mathrm{Li}$ and Be abundances of our sample stars. A difference in Be abundance somewhat larger than our errors is predicted between 1.7 and 4.5 Gyr: we do not find evidences for such a difference. Furthermore, between 6000 and $5900 \mathrm{~K}$ these models predict that a dispersion in $\log n(\mathrm{Li})$ of $\sim 0.6$ dex should correspond to a dispersion in $\log n(\mathrm{Be})$ of $\sim 0.2 \mathrm{dex}$, which is almost a factor of two larger than our errors. Stars S1252 and S1256 in M 67 show 0.75 dex difference in Li, but have virtually the same Be abundance, in 
contrast with the predictions. Even without considering the derived abundances, this result is evidenced by the comparison of the spectra of the two stars in the Be and Li regions shown in Fig. 1.

Gravity waves induced mixing predicts no significant Be depletion at 1.7 Gyr and only a small amount of depletion $(\sim 0.15$ dex $)$ for the coolest stars at the solar age. The predictions of gravity waves would be in contradiction with our results if hypothesis a) above is true and the sample stars have undergone some Be depletion; the curves showing the predictions of these models in panel a) indeed do not well fit the observed distribution. On the contrary, in panel b) models including waves provide the best fit to the observed Be vs. $T_{\text {eff }}$ diagram of our sample stars. Mixing driven by waves also seems to reproduce fairly well the Li vs. temperature distribution of IC 4651 stars, but is not able to reproduce the $\mathrm{Li}$ vs. temperature morphology of the older M 67 shown in Fig. 7. In particular, available models cannot explain the dispersion in Li observed in M 67. Rotating models including gravity waves are being developed (Montalbán \& Schatzman 2000); whereas, different rotation rates could provide an explanation for the dispersion in Li, preliminary qualitative analysis indicates that high rotation would result in lower predicted Li depletion thus not reproducing the lower envelope of M 67.

We finally mention that we have not taken into account in this discussion Li depletion due to mass loss. Models including mass loss (Swenson \& Faulkner 1992) predict that Be depletion starts only when Li has been completely depleted and thus they would be qualitatively in agreement with our results. Mass loss, however, has been convincingly shown not to work when applied to Li data alone (Swenson \& Faulkner 1992).

\section{Conclusions}

We have obtained the first Be measurements of late F/early-G dwarfs in old open clusters. The Be abundances of old cluster stars are compared with the abundance of a young Li-undepleted star and with the solar abundance: we find that all the sample stars share, within the errors the same Be abundance, irrespective of their age and $\mathrm{Li}$ abundance. This in turn suggests that these stars, including the Sun, do not undergo any Be depletion during the main sequence, unless the very unlikely hypothesis is accepted that they deplete Be, but do not deplete Li in the PMS phases. Our data are thus indicative of a shallow mixing process that extends deep enough in the stellar interior for Li burning to occur, but not deep enough to destroy Be. At variance with the results for field stars, we do not find any evidence of correlated $\mathrm{Li}$ and Be depletion, which would support shallow mixing driven by rotation. More in general, our observations and the comparison with various models show that the understanding of the mechanism regulating the boundary layers between the convective zone and the radiative core is still not satisfactory and that the problem of depletion of light elements during the MS phases of late F/early G-type stars, including our own Sun, needs further investigation on theoretical grounds. None of the models that we have discussed is able to simultaneously fit the observed Be vs. $T_{\text {eff }}$ and Li vs. $T_{\text {eff }}$ distributions of our sample stars and in particular their relative amounts of $\mathrm{Li}$ and Be depletion. Our sample stars cover a relatively small interval of effective temperatures; therefore we cannot extend our results/conclusions to warmer/cooler stars. Indeed, different mixing mechanisms may be at work in stars much warmer/cooler than the Sun, with much thinner/thicker convective zones. In particular, we note that the possibility that the mixing processes at work in F and G-type stars are different is not unrealistic since the transition between G- and F-type stars corresponds to the region in the HR diagram where several changes occur; namely, the surface convective zones gets thinner in F-type stars, possibly magnetic braking is not (or less) effective, and stars can retain high rotational velocities during their permanence on the main sequence. Most obviously, additional observations should carried out covering a larger temperature range.

Acknowledgements. We are grateful to Josefina Montalbán for sending us the prediction of models including gravity waves. This research was partially supported by MURST grants to $\mathrm{SR}$ and RP.

\section{References}

Allende Prieto, C., \& Lambert, D. L. 2000, AJ, 119, 2445

Anders, E., \& Grevesse, N. 1989, Geochim. Cosmochim. Acta, 53,197

Anthony-Twarog, B. J., Mukherjee, K., \& Caldwell, N., Twarog, B. 1998, AJ, 95, 1453

Balachandran, Suchitra C., \& Bell, R. A. 1998, Nature, 392, 791

Boesgaard, A. M., \& Budge, K. G. 1989, ApJ, 338, 875

Boesgaard, A. M., Deliyannis, C. P., King, J. R., et al. 1999, ApJ, 117, 1549

Boesgaard, A. M., \& King., J. R. 2002, ApJ, 565, 587

Boesgaard, A. M., Deliyannis, C. P., King., J. R., \& Stephens, A. 2001, ApJ, 553, 754

Chaboyer, B., Demarque, P., \& Pinsonneault, M. H. 1995, ApJ, 441,865

Chmielewski, Y., Müller, E. A., \& Brault, J. W. 1975, A\&A, 42,37

Deliyannis, C. P. 2000, in Stellar Clusters and Associations: Convection, Rotation, and Dynamos, ed. R. Pallavicini, G. Micela, \& S. Sciortino, ASP Conf. Ser., 198, 235

Deliyannis, C. P., \& Pinsonneault, M. 1997, ApJ, 488, 833

Deliyannis, C. P., Boesgaard, A. M., Stephens, A., et al. 1998, ApJ, 498, L147

Dekker, H., D' Odorico, S., Kaufer, A., Delabre, B., \& Kotzslowski, H. 2000, Proc. SPIE, 4008, 534

Eggen, O. J. 1971, ApJ, 166, 87

García López, R. J., Rebolo, R., \& Beckmann, J. E. 1988, PASP, 100, 1489

García López, R. J., Rebolo, R., \& Perez de Taoro, M. R. 1995, A\&A, 302, 184 
Jeffries, R. J., 2000, in Stellar Clusters and Associations: Convection, Rotation, and Dynamos, ed. R. Pallavicini, G. Micela, \& S. Sciortino, ASP Conf. Ser., 198, 245

Jones, B. F., Fisher, D., \& Soderblom, D. R. 1999, AJ, 117, 330

King, J. R., Deliyannis, C. P., \& Boesgaard, A. M. 1997, ApJ, 478,778

Kurucz, R. L. 1993, CD-ROMs \#1, 13, 18

Kurucz, R. L., Furenlid, I., Brault, J., \& Testerman, L. 1984, Solar Flux Atlas from 296 to 1300 nm, NOAO Atlas, No. 1

Montalbán, J., \& Schatzmann, E. 2000, A\&A, 354, 943

Pasquini, L. 2000, in The Light Elements and their Evolution, ed. L. da Silva, M. Spite, \& J. R. de Medeiros, IAU Symp. 198,245

Pasquini, L., Randich, S., \& Pallavicini, R. 1997, A\&A, 325, 535

Piau, L., \& Turck-Chieze, S. 2002, ApJ, 566, 419
Pinsonneault, M. H. 1997, ARA\&A, 35, 557

Primas, F., Duncan, D. K., Pinsonneault, M. H., Deliyannis, C. P., \& Thorburn, J. A. 1997, ApJ, 480, 784

Primas, F., Asplund, M., Nissen, P. E., \& Hill, V. 2001, A\&A, 364, L42

Randich, S., Pasquini, L., \& Pallavicini, R. 2000, A\&A, 356, L29

Randich, S., Pallavicini, R., Meola, G., Stauffer, J. R., \& Balachandran, S. C. 2001, A\&A, 372, 862

Sanders, W. L. 1977, A\&AS, 27, 89

Spite, F., Spite, M., Peterson, R. C., \& Chaffee, F. H. Jr. 1987, A\&A, 171, L8

Soderblom, D. R., Jones, B. F., Balachandran, S., et al. 1993, AJ, 106, 1059

Stephens, A., Boesgaard, A. M., King, J. R., \& Deliyannis, C. P. 1997, ApJ, 491, 339

Swenson, F. J., \& Faulkner, J. 1992, ApJ, 395, 654 